\title{
This paper is an artefact: On open science practices in design science research using registered reports
}

\author{
Cathal Doyle \\ Victoria University of Wellington \\ cathal.doyle@vuw.ac.nz
}

\author{
Markus Luczak-Roesch \\ Victoria University of Wellington \\ markus.luczak-roesch@vuw.ac.nz
}

\begin{abstract}
Design Science Research has not seen wide adoption of open science principles and practices so far. Here we investigate the use of registered reports, a functionality provided by the Open Science Framework online platform, for Design Science Research. We take an unconventional approach to develop a novel open Design Science Research process by instantiating the proposed process as a proof-of-concept of itself. This paper, therefore, becomes an artefact of this new open Design Science Research process itself and is structured accordingly. We make three contributions: (1) an innovative open Design Science Research process that can be executed using the Open Science Framework based on a registered reports template we developed; (2) a discussion how open Design Science Research is theoretically embedded in the field; and (3) a research agenda for the further development and evaluation of this novel approach to Design Science Research.
\end{abstract}

\section{Introduction}

Registered reports (RR) are a form of research artefact depositing and peer review in scientific research, with the aim of improving the transparency and reproducibility of the entire research life cycle, from the very early stages of the process up to the final publication of results. RR are a functionality provided by the Open Science Framework (OSF) online platform, and allow researchers to persistently and reliably (i.e. equipped with an persistent identifier and timestamp) pre-register planned studies. For a pre-registration, scholars can submit as much detail as they wish about their research idea and research design to get feedback from peers before collecting and analysing any data. In some fields academic journals formally require very detailed pre-registrations before scholars can even express a statement of intent to submit a research article to the journal for publication. In return these journals then offer those authors a pro-forma acceptance of their manuscript conditional on the research being carried out in accordance with the submitted RR and written up at adequate academic standard. Because OSF and RR are not focused on any particular scientific discipline it seems reasonable to trial the use of this particular approach to open science in a variety of areas, and investigate thoroughly how well it aligns with the traditional principles and practices scholars in these disciplines follow to date or alternative approaches to more transparent and reproducible scholarship.

Here we turn to Design Science Research (DSR) as one of the areas that has not seen wide adoption of open science principles and practices so far [4]. DSR is an approach to solve highly complex real world problems - also known as wicked problems - through the systematic development and evaluation of artefacts. Such artefacts can consist of constructs, models, methods, instantiations, design principles, technological rules, and/or design theories.

We contribute an innovative open DSR process with an accompanying $\mathrm{RR}$ template that allows researchers to execute their DSR projects following an open science approach. We also discuss along the lines of existing DSR theory as well as literature on open science principles and practices in other scientific domains if and why the open science approach of RR can be beneficial when conducting DSR. Finally, we lay out a research agenda for the next crucial steps to further develop and evaluate the idea of open DSR.

\section{Approach}

The scientific process in general (independent from whether open or not) and DSR in particular can be carried out in a multitude of ways with various opinions about what's right or wrong and very domain specific characteristics (e.g. a rigorous study in biomedical research can take a completely different form from valid and sound inquiry in computer science) $[1,3,19]$. In DSR this is evident 
by the numerous DSR process models that have been proposed over the years and where there is still no academic consensus about any particular process model being the canonical approach to DSR. We, therefore, suggest that our investigation into open science principles and practices for DSR can be regarded as a wicked problem that lends itself well to be addressed following a DSR approach.

In particular, we follow the DSR process model proposed by Peffers et al. [15] with the adjustment that we lift the entire process to align with the open science principles and practices of the OSF and RR. As a consequence of choosing the adapted open DSR approach this paper itself becomes part of an open DSR process and has been structured in an unconventional but innovative way that can be regarded as a blueprint for future studies that may follow our proposed open DSR approach.

We first give an overview of the OSF, explaining the terminology and different concepts involved, such as projects, preprints, and registered reports. Afterwards, the paper follows the structure of our open DSR project as it is represented on the OSF. Each component and sub-component is introduced together with a detailed explanation of the role of the OSF in the process of depositing and sharing artefacts and collecting open peer-reviews. For each component and sub-component we provide the persistent digital object identifier (DOI) that allows to retrieve the respective artefact as it has been deposited on OSF, where it is also possible for anyone to then publicly comment on the artefacts. We then describe how we follow the reflective practitioner approach [18] to formalise the experiences from this project, into an open DSR process and a template for creating RR for DSR projects.

\section{Overview of the Open Science Framework}

The Centre for Open Science (COS) is a nonprofit technology organisation with a mission to "increase openness, integrity, and reproducibility of research". To help achieve this they released an open source project called the Open Science Framework (OSF), available at https://osf.io, which facilitates open collaboration in scientific research. The OSF allows researchers to manage research projects, share preprints of their articles, and create registered reports.

On the OSF, a project is the highest level of categorisation that is supported, and can come in many forms such as experiments, lab groups, and/or papers. When setting up this top-level project, various details can be added including title, contributors, description, license, and tags. Projects are private by default, but can be made public for others to access, view and comment on. When projects are made public, researchers can obtain a Digital Object Identifier (DOI), which is a persistent link to the location of the project on the World Wide Web. This allows researchers to be able to easily share their projects, get feedback from others, or make findings openly available. Projects also contain a wiki, and allow for files to be uploaded to them.

Top-level projects are made up of different components, which are considered sub-projects within the top-level project. Categories are used to classify the types of components that can be added and include: "Project"; "Data"; "Hypothesis"; "Analysis"; "Communication"; "Instrumentation"; "Methods and Measures"; "Procedure"; "Software"; "Other"; or "Uncategorised". As such, components can be used to organise the research project by adding as many as needed, creating a hierarchy within the parent project. Each component also contains a wiki, and allows for files to be uploaded. Privacy settings, contributors, tags, wikis, and files, of components are separate from the parent project, but contributors and tags can be inherited. As such, a parent project can be made public while keeping its components private, and vice versa. Further, you can add nested components to components, which we refer to as sub-components.

The traditional route to disseminating research papers has been through academic journals or conferences that act as gatekeepers through their peer review and copy editing processes [13]. However, with the advent of the Internet and digital technologies, researchers can take much more control of the dissemination of their academic outputs themselves [8, 13]. It is becoming more and more common that researchers upload versions of their research outputs to specialised preprint servers such as OSF Preprint or arXiv, making outputs publicly available for others to view even before these works underwent traditional peer review [8]. This allows researchers to quickly disseminate information to the research community and receive feedback that can be implemented to improve the work before submitting to a journal or conference [13].

When uploading a preprint to the OSF, the first step for researchers is to choose what server they would like to upload it to. Once selected, researchers upload their file, and then enter information such as the license, a DOI if the article already has been accepted as a peer-reviewed publication, keywords, and an abstract. Researchers can also add the 
discipline and sub discipline the paper belongs to, and the authors involved. Lastly, researchers have the option to add supplementary materials by connecting the preprint with a top-level project, or one of its components/sub-components. Once submitted, the preprint is publically available with all the information from above visible, including the uploaded file. A DOI is also created for the preprint. Researchers can then update this information if necessary, and if they make changes to their file, version control is implemented, where anyone can view current and past versions.

Registered reports are a form of peer review that breaks studies into two stages $[8,13]$. The first stage, where authors pre-register their study, consists of the study rationale, research question(s), and the research methodology $[8,13]$. This is then submitted for peer review before any data is collected [8]. Feedback can then be provided to help improve the study rationale and ensure good methodological standards [13]. Once this has been completed, the second stage begins, where the study design is executed, data is collected and analysed, and the findings are reported [8]. Originally introduced in 2013 by the journal Cortex, RR are still mainly adopted in medical and psychological research [8], but the benefits are beginning to attract other fields.

On the OSF, a RR is a registration that creates a frozen, time-stamped copy of an OSF project. Thus, it can be used to capture snapshots of projects at different points in the research cycle, but in particular in the early stages where researchers pre-register research by submitting developed ideas and study designs. The OSF refers to these steps as "Develop Idea" and "Design Study" (https://osf.io/rr/). Once this stage has been peer reviewed, researchers move on to the second stage, where they collect and analyse the data, and write the report, where a second $\mathrm{RR}$ is created. The OSF refers to these steps as "Collect \& Analyse Data" and "Write Report" (https://osf.io/rr/). Researchers can make their RR publically available or embargo them for up to four years. However, RRs must eventually be made public. Further, the OSF allows many types of studies to be pre-registered, such as OSF Preregistration, Open-Ended Registration, and OSF-Standard PreData Collection Registration, all of which differ in the details they require authors to submit.

From this point onwards the structure of this paper follows that of our project on the OSF. We therefore recommend opening the project at https://www.doi.org/10.17605/osf.io/3hgq9.

\section{Top-Level Project: On open science practices in design science research}

On the OSF, one of the first steps that researchers can complete is that of creating a top-level project. We created a project titled "On open science practices in design science research", where we are investigating how to best align open science principles and practices with design science research. Figure 1 shows the details we provided for our toplevel DSR project on the OSF. This includes the project title, the two contributors, the category set as project, a description to inform others what the project is about, and the license type. Further to this, we added a number of tags that are not shown here but include "design science research", "open access", "open artefact", "open data", "open peer review", "open science", "open source", and "registered reports". Lastly, we made the project public, and created a DOI for others to be able to access, view, and leave comments on this project here: https://www.doi.org/10.17605/osf.io/3hgq9.

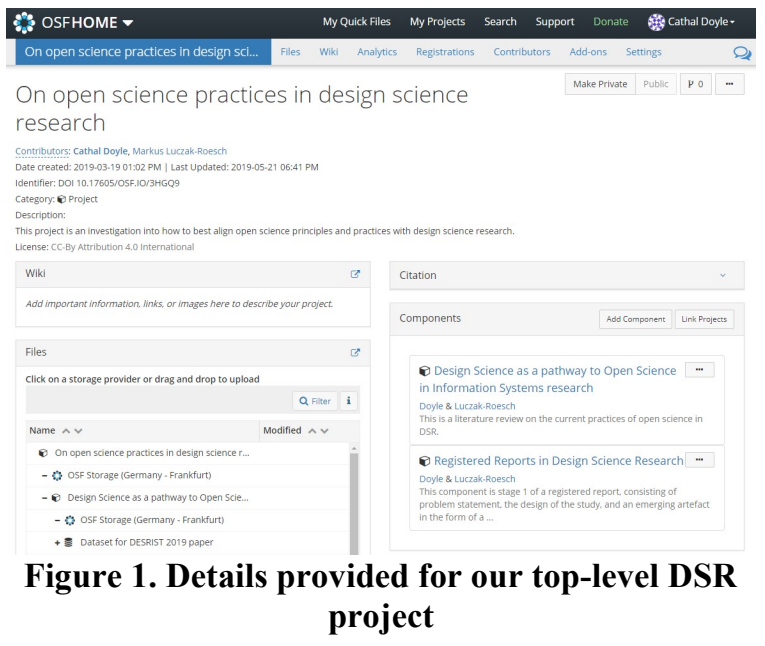

\subsection{Reflections on Top-Level Project}

To generalise the learning from this initial step, our understanding is that DSR researchers should create a top-level project for their DSR study when using the OSF to conduct research. In doing so they are creating the top entity of their hierarchical project, which will be the parent to each component added afterwards. There are a number of elements that must be added such as the title of the DSR project, and the contributors who are involved. There are some elements that are not required but we recommend should be added including setting the category to project, choosing an appropriate license 
to inform others of how the research can be used, and appropriate tags to describe the study so others can find it easier. Lastly, researchers can decide to set the privacy to private or public (this can be changed later also). Presented in Figure 2 is the first step for a method in creating registered reports for DSR projects, and the elements that should be added.

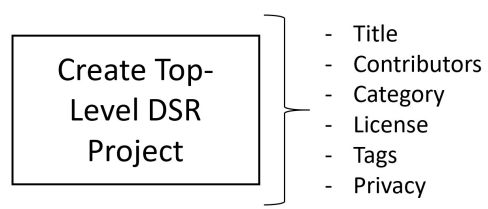

Figure 2. The first step to using registered reports in DSR projects

\subsection{Component 1: Design Science as a pathway to Open Science in Information Systems research}

The first component we created was a sub-project titled "Design science as a pathway to Open science in Information Systems research" which is a child to our top-level project. In this project, the aim was to "stimulate a discussion amongst IS scholars in general and DSR scholars in particular about how open science practices could be adopted within the field, and whether this is a necessary step forward to keep the pace of the changing academic environment." [4, pp.47]. The details provided for this component include a title, the authors involved, the category set as project, a description, and the license type, while you can access, view, and leave comments on this component here: https://www.doi.org/10.17605/osf.io/7dkrh.

While these details are similar to the top-level project, there are two noticeable differences. The first is that there is now a path created to indicate that this component sits within the top-level project. The second is that there is also a preprint of the paper we published at DESRIST 2019 from the study. This is linked to this component as it contains supplemental materials for the paper (you can access, view, and leave comments on this preprint here: https://doi.org/10.31219/osf.io/ye6xp). These supplementary materials are in the form of three subcomponents: the first is the dataset used for the paper; the second is the artefact that was built in it; and the third is the presentation slides from DESRIST 2019. This allows anybody who is viewing the preprint to review the supplementary materials (and have access to the DSR artefact), or anyone viewing this component to read the preprint. The first subcomponent is introduced next.

\subsubsection{Sub-Component 1: Dataset for DESRIST}

2019 Paper. In order to understand if open science (OS) was being practiced in DSR, we conducted a literature review of DSR papers. We focused on literature published in the senior scholar basket of eight journals, and the design science conference DESRIST, and applied an open science lense to them to see how many discussed, or practiced, open science. This resulted in a dataset of 479 articles, and we built a concept matrix to identify the ones that mentioned any of the OS components of open access, open data, open source, or open peer review. We made this dataset openly available for others to view, download, and use in their own studies by creating a sub-component for this project titled "Dataset for DESRIST 2019 Paper". This can further be used to confirm the findings in our study, and makes our claims more transparent. You can access, view, and leave comments on this sub-component here:

https://www.doi.org/10.17605/osf.io/hy5pe.

4.2.2. Sub-Component 2: OS DSR Process Model. A second sub-component was also made available, in the form of the framework that was designed and built for the DESRIST 2019 paper. This is an OS DSR process model, where the OS concepts are mapped to a generic DSR process model consisting of "Problem Identification", "Design and Build", "Evaluation", and "Communication". We refer to this as an open artefact, which was an emerging open science concept we identified from our literature review, that indicates how researchers can make their artefact(s) openly available for use by other academics and practitioners outside of reading the publications. The framework itself provides an understanding of how researchers conducting DSR can implement the different open science concepts. This is the first design cycle of this framework, where it has been designed and built but not yet evaluated. As shown in Figure 3, anyone can download and use the framework, and it is also evident that it is version 1 . When evaluated, we'll be able to add this to this sub-component, where we can show the full design cycle. Further, we'll be able to update the framework with the new learnings, and again make it openly available. This will provide a versioned history of the artefact, where a history of the changes will be visible. You can access, view, and leave comments on this sub-component here: https://www.doi.org/10.17605/osf.io/g6yj4 
OS DSR Process Model (Version: 1)

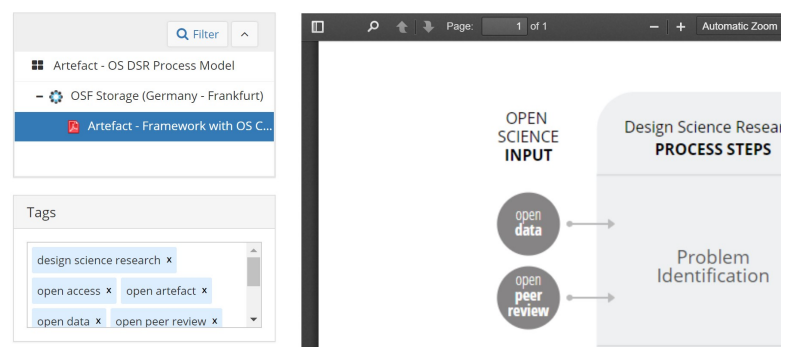

Figure 3. Overview of sub-component 2: OS DSR process model

4.2.3. Sub-Component 3: DESRIST 2019 Presentation. The third sub-component made available were the slides from the presentation of our paper at DESRIST 2019. This was made available before we presented so others could access them as we presented, to try and stimulate further discussion. It also allows others to access them after the conference in case they need to be reminded of something we said. The OSF can display the slides while viewers can also download them. You can access, view, and leave comments on this subcomponent here:

http://doi.org/10.17605/osf.io/9nkbs.

4.2.4. Reflections on Component 1. In terms of formalising the learning from this stage of the project, there are a few generalisations that can be made. An important aspect of any DSR project is looking at the knowledge base to inform both the problem identification, and/or the building of the artefact(s) (cite). This can consist of a literature review, as was done above, which can then be represented on the OSF through components and subcomponents. For example, a component can represent a stage in the DSR project (in this case the literature review), and then use sub-components to represent different elements of the literature review. In doing so, researchers can make these different elements of their review openly available, such as the dataset they used, and the artefacts they created (referred to as an open artefact [4]). This can result in researchers being more transparent about their literature reviews, allowing others to evaluate what was done, replicate the review if they want, and reproduce the same (or similar results), and/or confirm if these results are accurate. Further, researchers can make a preprint of any of their articles available, and link it to their component here. Once the researchers make their component public, they can encourage others to review their project, and their sub-components, to receive feedback. This allows for different elements to be updated, and as the OSF keeps a copy of each version of components and sub-components, a history of how they each evolve is provided.

\subsection{Component 2: Registered Reports in Design Science Research}

The second component we created is a project titled "Registered Reports in Design Science Research" which again sits as a sub-project to our top-level project, while it sits alongside our other sub-project. In this project, the focus is on registered reports in DSR, with the aim to understand how they can be adopted by researchers for their DSR projects. This was informed by our other sub-project, where it became apparent that little attention in terms of research has been paid to this area. As with all the other components, details like title, category, description, etc. need to be provided. You can access, view, and leave comments for this component here: https://www.doi.org/10.17605/osf.io/ktwqd

Further, there are four of sub-components for this project. As we will be creating a registered report, there are two required sub-components as outlined earlier. The first is the problem identification for this study, which includes the rationale and the problem statement. The second is the study design, which includes the data collection and data analysis steps. Additionally, we built two further artefacts: one is a registered report template for DSR, and the other is a method for creating registered reports for DSR projects. Each of these sub-components are introduced and explained over the following sections, beginning with the problem identification.

\subsubsection{Sub-Component 1: Problem Identification.} This sub-component is a requirement for RR, where it is part of the study preregistration. While in the OSF framework it is described as "Develop Idea", this maps well to the DSR concept of problem identification, which consists of developing the rationale for the study, and making a problem statement which helps to stimulate the research effort of the researcher(s) and the intended audience [15, 18]. Such a step is a feature in a number of DSR process models that researchers have developed for conducting DSR $[10,15,18]$. We have written and shared the problem identification as a PDF and made it available as a sub-component on the OSF, where others can make comments to help improve it. Our problem statement was written as "There is a lack of understanding of how registered reports can be adopted for DSR projects", where the objective of the study is to provide such an understanding. You can 
access, view, and leave comments on this subcomponent here:

https://www.doi.org/10.17605/osf.io/a8qy2.

4.3.2. Sub-Component 2: Study Design. This subcomponent is also a requirement for preregistration of a study in RR. The researchers set the protocol for how data is going to be gathered, and analysed. In doing so, others can review the study design and provide feedback before the researchers go out and collect the data. For this study, we wish to collect data in the form of feedback provided by IS researchers on our registered report, which includes the problem identification, the study design, and the two open artefacts we introduce further down (a registered report template for DSR and a method for creating registered reports for DSR projects). Similar to the problem identification, we have written and shared the study design as a PDF and made it available as a sub-component on the OSF. Included in the study design is an overview of the potential participants, how they'll be recruited, the method of data collection, and how the data will be analysed. Further to this, as we require human ethics approval to conduct the study, we created a HEC application which is also available for review here. You can access, view, and leave comments on this sumcomponent here:

https://www.doi.org/10.17605/osf.io/a7r5c.

\subsubsection{Sub-Component 3: Registered Report}

Template for DSR. The third sub-component of this project consists of an open artefact in the form of a template that could be used for creating RR for DSR. This emerged as we tried to register our project as a RR. As mentioned earlier, there are many different types of RR on the OSF, each with their own template, but most are geared towards other disciplines. For example, the template called "OSF Preregistration" requires a title and description but also hypotheses, which not all DSR projects will have, while the "OSF-Standard Pre-Data Collection Registration" only requires optional answers to two questions around whether data collection has begun, and if the researcher has looked at the data (there is also an other comments section). The only template that potentially suits DSR is "Open-Ended Registration" as it just has an optional summary, where DSR researchers can add in their problem identification and study design elements, however this isn't really of much use as researchers can avoid explaining the different elements of their DSR project. So, as one of the benefits of preregistration is to specify the plan of your study, it would be more useful if there was a template that DSR researchers could apply.

To design and build such a template, we adopted some of the required fields on the OSF.io templates (study title and authors), and added some important elements that DSR projects consist of (especially where we see these trends across DSR articles). Version 1 of the DSR RR template is presented in Table 1, which consists of required and optional fields. Those that are required include the problem identification (as many DSR process models require it); stating whether the evaluation being done is artificial or naturalistic (or both) [20]; and the impact stage of the study, i.e. proof-of-concept, proof-ofvalue, or proof-of-use [14].

Optional elements include stating the DSR process model that is going to be adopted (if one is being applied) as is done in many DSR publications; description of the outcomes for each step in the adopted process model as done by [5,9]; and to provide a description of the $\operatorname{artefact}(\mathrm{s})$ that will be built in the study. For this study, we used this template when creating the RR which will be discussed next. You can access, view, and leave comments on this sub-component here: https://www.doi.org/10.17605/osf.io/wxc8z.

Table 1. Registered Report Template for DSR

\begin{tabular}{|l|l|}
\hline Title & Description \\
\hline $\begin{array}{l}\text { Study Title } \\
\text { (required) }\end{array}$ & $\begin{array}{l}\text { Provide the working title of your } \\
\text { study. }\end{array}$ \\
\hline $\begin{array}{l}\text { Author(s) } \\
\text { (required) }\end{array}$ & $\begin{array}{l}\text { Add the authors involved in your } \\
\text { study. }\end{array}$ \\
\hline $\begin{array}{l}\text { Problem } \\
\text { identification } \\
\text { (optional) }\end{array}$ & $\begin{array}{l}\text { Explain the rationale of your study, } \\
\text { and state the problem (not needed if } \\
\text { you add this to the description of } \\
\text { your process model steps below). }\end{array}$ \\
\hline $\begin{array}{l}\text { DSR Process } \\
\text { model(s) } \\
\text { applied } \\
\text { (optional) }\end{array}$ & $\begin{array}{l}\text { State the DSR process model(s) that } \\
\text { will apply to conduct your study. }\end{array}$ \\
\hline $\begin{array}{l}\text { Describe } \\
\text { process } \\
\text { model steps } \\
\text { (optional) }\end{array}$ & $\begin{array}{l}\text { Describe the outcomes of your } \\
\text { project for each step(s) applied from } \\
\text { the process model. }\end{array}$ \\
\hline $\begin{array}{l}\text { Artefact(s) } \\
\text { (optional) }\end{array}$ & $\begin{array}{l}\text { Describe the artefact(s) that will be } \\
\text { developed in your study. }\end{array}$ \\
\hline $\begin{array}{l}\text { Evaluation } \\
\text { Type } \\
\text { (required) }\end{array}$ & $\begin{array}{l}\text { Artificial evaluation (explores the } \\
\text { performance of a solution in an } \\
\text { artificial setting such as laboratory } \\
\text { experiments, field experiments, } \\
\text { simulations, criteria-based analysis, } \\
\text { theoretical arguments, and/or } \\
\text { mathematical proofs) or naturalistic } \\
\text { evaluation }\end{array}$ \\
\hline
\end{tabular}




\begin{tabular}{|l|l|}
\hline & $\begin{array}{l}\text { performance of a solution in its real } \\
\text { environment i.e., within the } \\
\text { organization, such as case studies, } \\
\text { field studies, surveys, ethnography, } \\
\text { phenomenology, hermeneutic } \\
\text { methods, and/or action research) [20] } \\
\text { or both (explores the performance of } \\
\text { a solution in both an artificial and } \\
\text { naturalistic evaluation). }\end{array}$ \\
\hline Impact Stage & $\begin{array}{l}\text { The impact stage of your study is a } \\
\text { proof-of-concept (functional } \\
\text { feasibility of a potential solution) or } \\
\text { proof-of-value (investigate whether } \\
\text { stakeholders can use the solution to } \\
\text { create value across a variety of } \\
\text { contexts and conditions), or proof- } \\
\text { of-use (demonstrate that practitioners } \\
\text { can successfully create and gain } \\
\text { value from their own instances of the } \\
\text { generalizable solution) [14, pp.15]. }\end{array}$ \\
\hline
\end{tabular}

4.3.4. Registering the Report. For this study we created a registered report for our component 2, which is looking at RR in DSR, Under this component, as outlined above, are a number of subcomponents including the problem identification, the study design, and two open artefacts that were designed and built while conducting the study: a registered report template for DSR, and a method for creating RR for DSR projects which will be introduced next. RR create a snapshot of the project at this time, which cannot be altered afterwards. To create the RR, we went into component 2 and chose "Registrations". Here you add a new registration by choosing a template to follow - we chose "OpenEnded Registration" and in the "Summary" box we used the headings from our registered report template for DSR to complete the preregistration. RR contains information such as the summary, registration type, date registered, category, and the number of components, and comments, which viewers can then click to view. RR are immediately published once the project administrators confirm it, or they can set an embargo for up to 4 year. You can access, view, and leave comments on our RR here: https://osf.io/9g5au.

4.3.5. Reflections on Component 2. Formalising the learning at this stage of the project, there are a number of generalisations that can be made, as well as further reflections from the first component. While the first component highlighted a way to be able to represent the step of accessing the knowledge base to inform the understanding of the problem through a literature review (providing the dataset, and open artefact that were created), this component highlights that researchers can use components to structure the different stages of their DSR projects. For example, if they are applying a DSR process model to conduct their study, each step in the model can be represented by a component. In doing so, researchers can build a "portfolio" of their project, and as it progresses, they can make each publically available. For example, researchers can create a component titled "Design, Build, and Evaluate" and have a sub-component for each of their design cycles titled "Design Cycle 1", "Design Cycle 2", etc.. In doing so, others would be able to see the evolution of the artefact from its conception through to completion. Once made public, and a DOI is assigned to this step, it can be added to any publication that talks about the artefact. This would be addressing an issue that can be observed in many published DSR articles where the researchers aren't very transparent with the evolution of the artefact, but mainly provide the completed artefact followed by the final evaluation of it. The DOI could be added to a sentence that explains if you wish to see the design cycles please go here. This can be applied to other common DSR process steps such as the problem identification (as represented above) and communication. Thus, components and subcomponents can consist of different elements of a DSR study such as DSR process steps, literature reviews, problem identification, study design, design cycles, datasets, open artefacts, presentations, human ethics applications, data analysis, and/or open peer reviews, as represented in Figure 4, where subcomponents are linked to components.

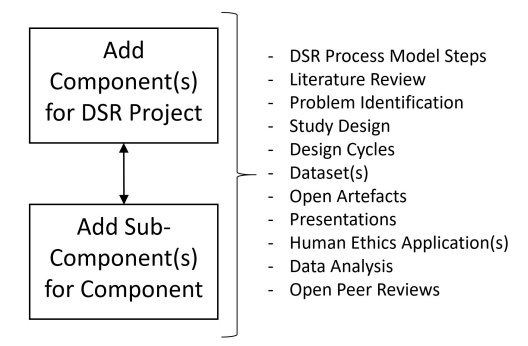

Figure 4. The second and third steps to using registered reports in DSR projects

A further generalisation is around utilising RR for DSR projects. While the default use for RR is to create a frozen, time-stamped copy of a project, where authors preregister their study, the OSF also provides an opportunity to create snapshots of components and sub-components at any time (as shown in Figure 5). This suggests that researchers can create such snapshots for different elements of their project, from the top-level project, to individual components within that project, down to subcomponents within those components. 


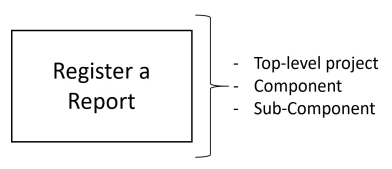

Figure 5. The fourth step to using registered reports in DSR projects

\subsubsection{Sub-Component 4: A method for creating} registered reports for DSR projects. The final subcomponent is an open artefact that consists of a method for creating RR in DSR. This method has been derived from the experience of developing a $R R$ for this study, and reflecting on the learning that occurred to make it more generalisable. The method is presented in Figure 6 and consists of four steps. The first requires creating a top-level DSR project, providing different details such as a title, contributors and license type. Once this is completed, researchers can add as many components as required to structure their DSR project, and this can consist of things such as literature reviews, the problem identification, study designs, etc. Researchers can then add subcomponents to these components, and these can again consist of the same elements as above, such as literature reviews, problem identification, etc. While the decision on how to best structure a DSR project on the OSF is up to the researchers as no two projects are alike, we would recommend that it might help to use the DSR process model that is being used to conduct the study. Lastly, researchers have the opportunity to register a report for any of these components, hence why it is represented as a boundary around them. Obviously, if researchers want to create a RR for journals they will be required to complete the standard process of preregistration, but they can still use them for other purposes also as described earlier. As there is no default RR template for DSR, we recommend that researchers use the template we provided in our sub-component 3 when creating a RR on the OSF (we will be suggesting to OSF that they adopt this template as it evolves).

\section{Discussion and Conclusion}

While the DSR field has matured over the last twenty years, it has been slow to develop, or adopt, computer-based tools to help researchers structure, document, manage, and present their projects $[2,12]$. The projects are often complex in nature, where researchers have to keep track of decisions made, while documenting their activities [2] including their design cycles. Thus, during the 2017 DESRIST conference, Morana et al. [12] held an open workshop with DSR researchers to help determine what they need from a tool to effectively and

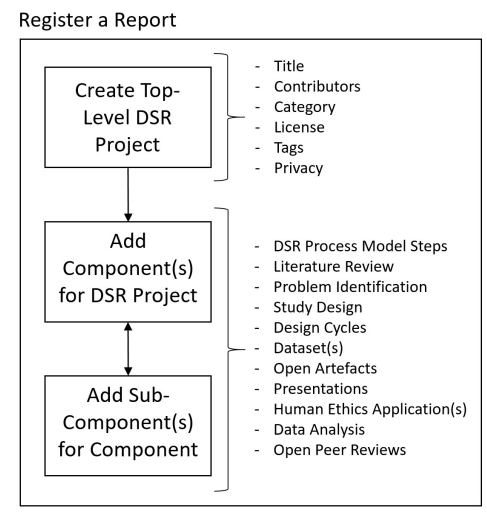

Figure 6. A method for creating registered reports for DSR projects

efficiently perform their work. This exercise resulted in three generic categories of requirements: predesign, design, and post-design, and nine requirements categories across them.

Our study posits that registered reports, and the Open Science Framework, can help to address a number of the aforementioned requirements. For example, in the pre-design stage, Morana et al. [9, pp.242] state that DSR researchers "need guidance in formulating research problems and in highlighting their relevance in terms of scholarly and practical utility". RR help address this requirement, where researchers share their problem identification to get feedback from other stakeholders, i.e. researchers, and practitioners, who can identify whether it's a problem worth solving, has already been solved, or whether the focus should be adjusted. Further, this can help to avoid type III errors, which occur when researchers use the right research method but provide answers to the wrong question(s) [16].

$\mathrm{RR}$ also highlight a requirement that was not identified in the pre-design stage by Morana et al. [12], namely the opportunity for open peer review of the study design, including how the artefact(s) will be evaluated. Since it is important that DSR researchers ensure the application of rigorous methods to construct and evaluate their artefacts [7], DSR researchers can make their research design available and have their peers critically evaluate the design and method, before it is executed. Such reviews can help improve the rigor of the research design (often an area of criticism for DSR research). It is our contention that RR can help produce more relevant and rigorous DSR by receiving feedback on both the problem to be investigated, and the research design, before running the project.

From the perspective of the OSF, it adheres to six of the seven design principles used to build DSR project support tools that were developed by van 
Brocke et al. [2]. Presented in Table 2 is an explanation of this adherence. However, we note a major OSF feature that van Brocke et al. [2] did not address, which is the ability to make DSR projects openly accessible to the public (either immediately or after an embargo period).

Table 2. OSF Implementation of DPs for DSR Project Support Tools

\section{Description}

The OSF currently does not offer specific DSR templates but it is a suggestion we propose in this study (see Table 1)

DP2 Context-sensitivity

The OSF allows users to customize their chosen DSR approach by allowing users to create components and sub-components to represent any stage in the DSR project.

\section{DP3 Design as an iterative process}

The OSF allows users to document the iterations of their design cycles by allowing users to create components to represent each design cycle, and allows for version control.

\section{DP4 Collaboration support}

The OSF allows users to collaborate across all components of their DSR projects by allowing project owners to add contributors.

\section{DP5 Knowledge extraction}

The OSF allows users to extract knowledge and document learning from their design DSR projects by allowing users to upload any document type to represent these activities.

\section{DP6 Communication support}

The OSF allows users to effectively communicate research results through preprints and sharing of DOIs to specific components and sub-components.

\section{DP7 Data security}

The OSF ensures data privacy and security of stored information by using bcrypt hashes for passwords, three types of hashes (MD5, SHA-1, SHA-256) for files, and SSL for data transfers.

While it is acknowledged that research outcomes should be made available for other researchers, reviewers, and practitioners [2], we suggest that DSR theory actually requires scholars to take this further by making as much of the research process openly available as possible (always ensuring that this happens without violating principles of ethical scholarship, confidentiality and good research data stewardship). We believe this would further address the call made by van Brocke et al. [2, pp.2], where such a tool will "ultimately increase collaboration, traceability, and quality in DSR".

\subsection{Open Design Science Research: An Agenda}

5.1.1. Towards Proof-of-Value and Proof-of-Use of Open Design Science Research. This study instantiated a first-of-its-kind open Design Science Research process as a proof-of-concept to understand if, and how, DSR can be adapted to incorporate the use of registered reports on the OSF. We encourage other researchers to now apply this new process to confirm its proof-of-value, and proof-of-use [14] when conducting DSR. In doing so we can begin to refine and improve this first iteration of the open DSR process to make it applicable to a wider variety of DSR projects. Further, it would be of value to adapt other DSR process models to the open science principles and practices of the OSF and RR. In doing so, we can start to develop a consensus about how RR and the OSF can be best applied to DSR. This should help increase the legitimacy of using open science, and RR as legitimate approaches to conduct DSR.

5.1.2. Breaking up the Peer Review Monolith. Peer review has been an important step in the dissemination of scientific knowledge [13] and can be defined as "the process by which new knowledge is legitimized by its acceptance and dissemination to the wider community" [10, pp.29]. In recent years, with the advancement of technology, more diverse evaluation processes have emerged $[11,13]$ in the form of pre- and post-publication peer review mechanisms [13]. As shown in this study, RR and the OSF implement such mechanisms, where different stakeholders can provide feedback on components, and sub-components. However, in recent years there has also been an increasing level of papers to be reviewed, putting high pressure on the academic community to meet the demand [11]. Thus, it is important that we start to consider how to improve the peer review process. RR may offer a solution by breaking the review process into two stages, where reviews are first done on the problem identification and study design, and then on the findings that are reported. This breakdown may help reduce the burden on reviewers, since poorly designed research will be filtered out before submission to an academic conference or journal.

5.1.3. Incentivising Open Peer Review. A number of initiatives have been launched in recent years to incentivize scholarly activity more holistically beyond measuring the pure quantity of publications and citations. Amongst those are open access platforms such as ScienceOpen 
(https://www.scienceopen.com/) [6], where academics can disseminate their personal open access publications and contribute to the quality assurance in their respective discipline by managing article collections, or Publons (https://publons.com) [17], which allows to record and verify peer reviews. The latter is an example of a system that makes peer reviews a recognised academic contribution not just as a pure counted quantity but also based on the quality of the actual content of written reviews [13]. These considerations also need to be addressed by the DSR community to move away from the traditional single- or double-blind evaluation processes into more open ones.

5.1.4. Overcoming the Research-Practice Paradox. One of the key aims of DSR has always been to increase the practice value of artefacts and the underlying research. However, the misalignment of the academic and business life cycles creates a paradoxical situation. DSR output artefacts are hardly ever made available before the actual publication of research results in an academic conference or journal (if they are made available at all). Because publishing can take up to years to happen, the community is fundamentally out of sync with the agility that is common in the business world. In order to overcome this paradox, we suggest that future work on open Design Science Research investigates the possibility of linking academic publishing platforms such as OSF with platforms used in IS/IT businesses such as Confluence or Github to stimulate cross sector adoption, adaptation and feedback (e.g. through the ability to fork an artefact from OSF to Github or to feed issues raised about an artefact on Github back to OSF).

\section{References}

[1] Barker, A., and J. Van Hemert, "Scientific workflow: a survey and research directions", International Conference on Parallel Processing and Applied Mathematics, Springer, 746-753.

[2] vom Brocke, J., P. Fettke, M. Gau, et al., "Tool-Support for Design Science Research: Design Principles and Instantiation", SSRN Electronic Journal, 2017.

[3] Cooper, J., J.O. Vik, and D. Waltemath, "A call for virtual experiments: accelerating the scientific process", Progress in biophysics and molecular biology 117(1), 2015, pp. 99-106.

[4] Doyle, C., M. Luczak-Roesch, and A. Mittal, "We Need the Open Artefact: Design Science as a Pathway to Open Science in Information Systems Research", International Conference on Design Science Research in Information Systems and Technology, Springer International Publishing (2019), 46-60.
[5] Gregor, S., A. Imran, and T. Turner, "A 'sweet spot'change strategy for a least developed country: leveraging e-Government in Bangladesh", European Journal of Information Systems 23(6), 2014, pp. 655-671.

[6] Grossmann, A., "Publishing in transition-do we still need scientific journals?", ScienceOpen Research, 2015.

[7] Hevner, A., S. March, J. Park, and S. Ram, "Design Science in Information Systems Research", MIS Quarterly 28(1), 2004, pp. 75-105.

[8] Horbach, S.S., and W.W. Halffman, "The changing forms and expectations of peer review", Research integrity and peer review 3(1), 2018, pp. 8.

[9] Hustad, E., and D.H. Olsen, "Educating Reflective Enterprise Systems Practitioners: A Design Research Study of the Iterative Building of a Teaching Framework", Information SYstems Journal 24(5), 2014, pp. 445-473.

[10] Kuechler, B., and V. Vaishnavi, "On Theory Development in Design Science Research: Anatomy of a Research Project", European Journal of Information Systems 17(5), 2008, pp. 489-504.

[11] Mandviwalla, M., R. Patnayakuni, and D. Schuff, "Improving the peer review process with information technology", Decision Support Systems 46(1), 2008, pp. 29-40.

[12] Morana, S., J. vom Brocke, A. Maedche, S. Seidel, and M.T.P. Adam, "Tool Support for Design Science Research-Towards a Sotware Ecosystem: A Report from a DESRIST 2017 Workshop", Workshop," Communications of the Association for Information Systems: Vol, 17.

[13] Munafò, M.R., B.A. Nosek, D.V.M. Bishop, et al., "A manifesto for reproducible science", Nature Human Behaviour 1(1), 2017, pp. 21.

[14] Nunamaker Jr, J.F., R.O. Briggs, D.C. Derrick, and G. Schwabe, "The last research mile: Achieving both rigor and relevance in information systems research", Journal of Management Information Systems 32(3), 2015, pp. 10-47.

[15] Peffers, K., T. Tuunanen, M.A. Rothenberger, and S. Chatterjee, "A Design Science Research Methodology for Information Systems Research", Journal of Management Information Systems 24(3), 2007, pp. 45-77.

[16] Rai, A., "Editor's comments: avoiding type III errors: formulating IS research problems that matter", MIS Quarterly 41(2), 2017, pp. iii-vii.

[17] Rajpert-De Meyts, E., S. Losito, and D.T. Carrell, "Rewarding peer-review work: the Publons initiative", Andrology 4(6), 2016, pp. 985-986.

[18] Sein, M., O. Henfridsson, S. Purao, M. Rossi, and R. Lindgren, "Action Design Research", MIS Quarterly 35(1), 2011, pp. 37-56.

[19] Vallance, P., A. Freeman, and M. Stewart, "Data sharing as part of the normal scientific process: a view from the pharmaceutical industry", PLoS medicine 13(1), 2016, pp. e1001936.

[20] Venable, J., J. Pries-Heje, and R. Baskerville, "A comprehensive framework for evaluation in design science research", International Conference on Design Science Research in Information Systems, Springer (2012), 423438. 